\title{
Preoperative Physician Fitness
}

\author{
Udas C Ghosh ${ }^{1}$, Kaushik Hazra ${ }^{2}$
}

\begin{abstract}
A preoperative checkup is necessary for the patients who are preparing for surgery. The opinion of the internist is vital to decide the suitability of major and minor surgeries by assessing the health status of patients. There are different key parameters to be monitored and recorded, which includes a proper history, thorough clinical examination and an appropriate laboratory checkup. By assessing pre and perioperative risks, the role of physician is to optimize patient's medical conditions to reduce morbidity and mortality. This article discusses these topics at length and provides some recommendations for fellow practitioners.

Keywords: General physicians, Laboratory checkup, Mortality, Preoperative.

Bengal Physician Journal (2019): 10.5005/jp-journals-10100-6105
\end{abstract}

\section{INTRODUCTION}

Adequate preoperative preparation and optimized treatment of preexisting diseases are very essential for a patient undergoing surgery to avoid peri and postoperative morbidity and or mortality and the person to take an active role to assess the possible complications during operation is a primary-care physician. So a systematic high-quality and cost-effective approach is needed for a safe surgery. ${ }^{1,2}$

These are the primary goals of a physician for a preoperative assessment: ${ }^{3}$

- To assess the overall health status of the patients.

- To detect undiagnosed clinical conditions.

- To detect a preoperative risk.

- To optimize patient's medical condition and to reduce hospital staying.

\section{General Health Assessment \\ History}

It is the most important component of preoperative evaluation. It includes thorough present, past, family, personal histories, drug history, including allergy to a drug (if any) to be taken. ${ }^{1-3}$

\section{Physical Examination}

It includes assessment of the airway, lungs, heart, and vital signs. Investigations are to be done for any significant finding in a physical examination. ${ }^{1,4}$

\section{Drug History}

A thorough drug history should be taken. Some drugs such as aspirin (at least 3 days before surgery), clopidogrel ( 7 days before surgery), and oral contraceptives ( 6 weeks before surgery) are to be stopped before surgery and some modification of dosage may be required such as antidiabetics and antihypertensive.

\section{Laboratory Workup}

Usually routine laboratory tests are not recommended for healthy individuals as a preoperative assessment. Depending upon the findings in clinical examination and history, laboratory tests are advised (Table 1).
${ }^{1}$ Department of Medicine, Murshidabad Medical College, Berhampopre, Murshidabad, West Bengal, India

${ }^{2}$ Department of Medicine, Jagannath Gupta Institute of Medical Sciences and Hospital, Budge Budge, West Bengal, India

Corresponding Author: Kaushik Hazra, Department of Medicine, Jagannath Gupta Institute of Medical Sciences and Hospital, Budge Budge, West Bengal, India, Phone:+91 9903073136, e-mail:drkaushik6@ gmail.com

How to cite this article: Ghosh UC, Hazra K. Preoperative Physician Fitness. Bengal Physician Journal 2019;6(1):17-20.

Source of support: Nil

Conflict of interest: None

Table 1: Indications of laboratory tests ${ }^{1,5}$

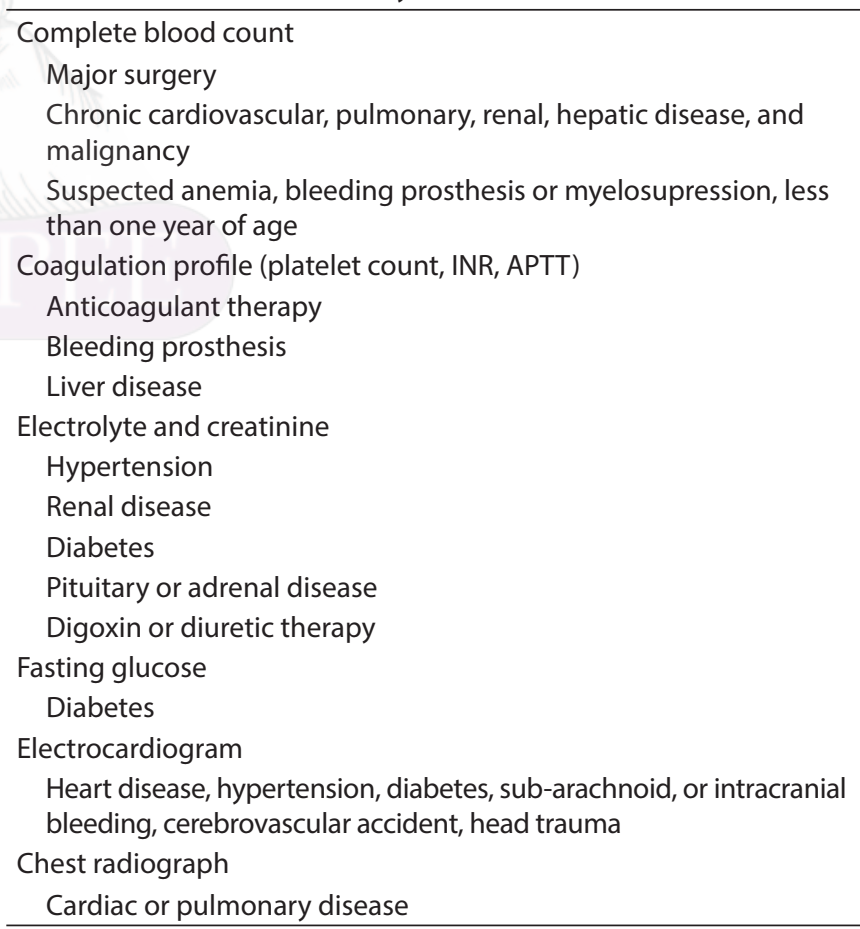

Source: Zambouri A. Preoperative evaluation and preparation for anesthesia and surgery. Hippokratia 2007;11(1):13-21

(c) The Author(s). 2019 Open Access This article is distributed under the terms of the Creative Commons Attribution 4.0 International License (https://creativecommons. org/licenses/by-nc/4.0/), which permits unrestricted use, distribution, and non-commercial reproduction in any medium, provided you give appropriate credit to the original author(s) and the source, provide a link to the Creative Commons license, and indicate if changes were made. The Creative Commons Public Domain Dedication waiver (http://creativecommons.org/publicdomain/zero/1.0/) applies to the data made available in this article, unless otherwise stated. 


\section{Complete Blood Count}

Usually hemoglobin and hematocrit measurement are required before surgery, especially in a case of anemia or prolonged surgery with anticipated significant blood loss. It is also indicated in some preexisting chronic diseases such as chronic kidney disease, chronic liver disease, or any chronic inflammatory condition. ${ }^{6,7}$

\section{Coagulation Testing}

Coagulation tests such as platelet count, PT, and APTT are usually done before any major surgery and also in patients with impaired hemostasis or patients taking anticoagulants or in patients with a spontaneous bleeding history or a known family history of coagulopathy. ${ }^{6-8}$

\section{Electrolyte and Creatinine Testing}

Indicated usually in patients of hypertension, heart failure, chronic kidney disease, chronic liver disease, or in patients taking drugs such as diuretics, angiotensin-converting enzyme inhibitors, angiotensin receptor blockers, nonsteroidal anti-inflammatory drugs, and digoxin.

According to few guidelines, serum electrolyte assessment is indicated in patients aged more than 40 years but usually not followed if history and physical examinations are normal. ${ }^{6,7}$

\section{Glucose and HbA1C Testing}

In known diabetics or patients on medication such as corticosteroid or in patients with a history suggestive of undiagnosed diabetes, glucose and A1C measurement are indicated. ${ }^{6-8}$

\section{Electrocardiography}

Patients should have preoperative ECG before a high-risk or intermediate-risk surgical procedure. ECG is usually not indicated in patients undergoing low-risk procedures. Cardiac risk factors for noncardiac surgery are assessed ${ }^{6-8}$ (Table 2).

\section{Chest Radiography ${ }^{6-9}$}

Usually not indicated routinely in healthy patients but recommended as a preoperative assessment in known pulmonary or cardiac disease. Abnormal chronic findings in chest X-ray do not alter the outcome of surgery; so previous chest X-ray reports should also be assessed.

These are the risk factors of perioperative pulmonary complications:

- Chronic obstructive pulmonary disease,

- Age older than 60 years,

- Functional dependence,

Table 2: Surgery related risk of perioperative cardiac complications

\begin{tabular}{ll}
\hline Risk of procedure & Examples \\
\hline High $(>5 \%)$ & Aortic and major vascular surgery, \\
& peripheral vascular surgery \\
Intermediate $(1-5 \%)$ & Intraperitoneal or intrathoracic \\
& surgery, carotid endarterectomy, \\
& head and neck surgery, orthopedic \\
& surgery, prostate surgery \\
& Ambulatory surgery, breast surgery, \\
Low $(<1 \%)$ & endoscopic procedures, superficial \\
& procedures, and cataract surgery \\
\hline
\end{tabular}

Source: Zambouri A. Preoperative evaluation and preparation for anesthesia and surgery. Hippokratia 2007;11(1):13-21
- Congestive heart failure,

- Emergency procedure,

- Prolonged procedures.

\section{Urinalysis}

Though routinely not recommended, it is suggested in patients undergoing surgical implantation of foreign material (like prosthetic joint, heart valve) or invasive urologic procedures. ${ }^{6,8}$

\section{Perioperative Risk Assessment ${ }^{1,10}$}

Depending upon the risk associated with a surgery, there are some grading system. Among them one is ASA (American Society of Anesthesiologists) grading system, which is very simple and correlates the risk of anesthesia and surgery with patient's general health and other comorbid conditions.

It is very useful and applied to almost all patients before surgery. Increased grading score is associated with increased pre- and perioperative complications, especially in ASA class 4 and 5 (Table 3 ).

\section{Assessment of Cardiovascular Risk ${ }^{1,11}$}

To assess the risk factors for noncardiac surgery, the American College of Cardiology (ACC) and the American Heart Association

Table 3: American Society of Anesthesiologists' classification of physical status

ASA class 1

No organic, physiological, biochemical or psychiatric disturbances ASA class 2

Mild-to-moderate systemic disturbance that may or may not be related to the reason of surgery (e.g. heart disease that only slightly limits physical activity, essential hypertension, diabetes mellitus, anemia, extremes of age, morbid obesity, and chronic bronchitis)

ASA class 3

Severe systemic disturbance that may or may not be related to the reason for surgery (does limit surgery), e.g. heart disease that limits activity, poorly controlled essential hypertension, diabetes mellitus with vascular complications, chronic pulmonary disease that limits activity, angina pectoris, and a history of prior myocardial infarction

ASA class 4

Severe systemic disturbance that is life-threatening with or without surgery-e.g. congestive heart failure, persistent angina pectoris, advanced pulmonary, renal, or hepatic dysfunction

ASA class 5

A moribund patient who has a little chance of survival but is submitted to surgery as a last resort (resuscitative effort)—e.g. uncontrolled hemorrhage as from a ruptured abdominal aneurysm, cerebral trauma, pulmonary embolus

ASA class 6

A declared brain-dead patient whose organs are being removed for donor purposes

E

An $E$ is added to the status number to designate an emergency operation

Source: Zambouri A. Preoperative evaluation and preparation for anesthesia and surgery. Hippokratia 2007;11(1):13-21 
(AHA) have published guidelines for perioperative cardiovascular evaluation for noncardiac surgery.

These are:

- Major clinical predictors (markers of unstable coronary artery disease):

- Myocardial infarction less than 6 weeks

- Unstable or severe angina (class 3-4)

- Decompensated congestive heart failure Significant arrhythmias (causing hemodynamic instability). Severe valvular disease (aortic/mitral stenosis with valve area less than $1 \mathrm{~cm}^{2}$ ). CABG /PTCA less than 6 weeks

- Intermediate clinical predictors (markers of unstable coronary artery disease):

- Previous myocardial infarction more than 6 weeks and less than 3 months based on a history or the presence of pathological q waves

- Mild angina (class 1-2)

- Silent ischemia, compensated congestive heart failure, ejection fraction less than $35 \%$, post CABG/PTCA more than 6 weeks and less than 3 months, or more than 6 years with antianginal therapy

- Diabetes mellitus

- Renal insufficiency

- Minor clinical predictors (increased probability of coronary artery disease):

- Family history of coronary artery disease

- Age more than 70 years

- ECG abnormalities (arrhythmias, LVH, LBBB)

- Low functional capacity

- A history of stroke

- Uncontrolled systemic hypertension

- Hypercholesterolemia, smoking

- Post infarction (more than 3 months), asymptomatic without treatment

- Post CABG/PTCA more than 3 months and less than 6 years and no symptoms of angina nor anti-anginal therapy

\section{Assessment of Pulmonary Risk}

Postoperative pulmonary complications are pneumonia, atelectasis, bronchitis, bronchospasm, and respiratory failure.

Risk factors are: ${ }^{1,12}$

- Depending upon the surgery:

Risk is greater in upper abdominal and thoracic surgery, prolonged surgery more than 3 hours, surgery under general anesthesia, and in emergency surgery.

- Known obstructive airway disease (asthma, COPD) patients:

All COPD or asthma patients with a history of hospitalization within last 6 months are at high risk before surgery.

- In other chronic lung diseases such as interstitial lung diseases.

- In known smoker patients.

- Elderly patients especially in age $>60$ years.

- Obese patients.

- Presence of obstructive sleep apnea.

- Poor general health status.

\section{Preventive Steps to Avoid these COMPLications COPD/Asthma}

For obstructive airway disease patients, the underlying conditions should be under controlled before surgery. There should be no wheezing and peak flow should be greater than $80 \%$ of predicted. If necessary, a short course of steroid (1 mg per kg body weight of prednisolone or other in equivalent doses) should be given. Patients with a recent history of hospitalization should be assessed more carefully as airway reactivity may persist for several weeks. COPD patients should be examined and investigated for corpulmonale. Impaired nutrition status and electrolyte imbalance should be corrected as they cause respiratory muscle weakness.

\section{Diabetes Mellitus ${ }^{1,13}$}

Diabetic patients are at greater risk than a nondiabetic as long-term complications of diabetic are more. There are some recommendations before surgery to prevent perioperative complications:

- Blood glucose should be maintained below $180 \mathrm{mg} / \mathrm{dL}$ until oral feeding is started as blood glucose more than $180 \mathrm{~g} / \mathrm{dL}(10 \mathrm{mmol} / \mathrm{L})$ causes osmotic diuresis, dehydrations, and other complications.

- OHA should be withheld on the day of surgery and may be stopped up to 48 hours after operation. To minimize the metabolic consequences of surgical stress, a combination of glucose and insulin should be given as per glycemic status. Usually in a $70 \mathrm{~kg}$ patient, 1 unit/hour regular insulin causes a decrease in blood glucose by approximately 25 to $30 \mathrm{mg} / \mathrm{dL}$. In minor surgery, usually glucose insulin combination is not required.

\section{Patients on Anticoagulation ${ }^{1,14}$}

It is very difficult to adjust the dosage of anticoagulation for a person undergoing surgery as a major concern is the increased risk of hemorrhage or thromboembolism after discontinuation of oral anticoagulation therapy. So there are some recommendations to avoid this complication:

- INR should be kept around 1.5.

- Oral anticoagulant to be stopped at least 4 days before surgery to reach the INR around 1.5 (if previous INR was 2-3), and after surgery it will take atleast 3 days to reach INR around 2 .

- INR measurement to be repeated one day prior to surgery and if it is more than $1.7,1 \mathrm{mg}$ vitamin $\mathrm{K}$ injection SC/IV to be given and if INR is more than 1.5 on the day of surgery, one or two units of FFP transfusion to be given before any major surgery.

\section{Patients Receiving Antiplatelet Drugs ${ }^{1}$}

According to The American Society of Regional Anesthesia (ASRA) and European Societies, there are some recommendations for these patients to avoid major life-threatening bleeding complications during surgery and these are:

- There should atleast be 3 days aspirin-free interval before surgery.

- Ticlopidine should bestopped at least 14 days before surgery.

- Clopidogrel should be stopped 7 days prior to surgery.

- Abciximab should be discontinued 48 hours before surgery.

- Eptifibatide and tirofiban should be stopped 8 hours before surgery. 


\section{Conclusion}

Assessment of a patient undergoing surgery is very important to avoid perioperative complications and the role of a physician is very crucial here. These are some recommendations of preoperative assessment and should be followed to decrease the morbidity and mortality of patients in significant amounts.

\section{References}

1. Zambouri A. Preoperative evaluation and preparation for anesthesia and surgery. Hippokratia 2007;11(1):13-21.

2. Roizen MF, Foss JF, et al. Preoperative evaluation in Anesthesia. 5th ed., Miller RD. ed., Philadelphia: Churchill-Livingstone; 2000. pp. 824-883.

3. Pedersen T, Eliasen $\mathrm{K}$, et al. A prospective study of mortality associated with anesthesia and surgery: risk indicators of mortality in hospital. Acta Anaesthesiol Scand 1990;34:176. DOI: 10.1111/j.1399-6576.1990. tb03066.x.

4. Kitts JB. The preoperative assessment; who is responsible? Can J Anesth 1997;44:1232-1236. DOI: 10.1007/BF03012768.

5. American Society of Anesthesiologists Task Force on Preanesthesia Evaluation. Practice Advisory for Preanesthesia Evaluation. Anesthesiology 2002;96:485-496. DOI: 10.1097/00000542-20020200000037.

6. Feely MA, Collins CS, et al. Preoperative testing before noncardiac surgery: Guidelines and Recommendations,. Am Fam Physician 2013 Mar 15;87(6):414-418.

7. Card R, Sawyer M, et al. Perioperative Protocol. Institute for Clinical Systems Improvement; Updated March 2014.
8. National Institute for Clinical Excellence. Preoperative tests: the use of routine preoperative tests for elective surgery. http://www.nice. org.uk/nicemedia/pdf/CG3NICEguideline.pdf. Accessed December 12, 2012.

9. Poldermans D, Bax JJ, et al. Task Force for Preoperative Cardiac Risk Assessment and Perioperative Cardiac Management in Noncardiac Surgery; European Society of Cardiology (ESC). Guidelines for pre-operative cardiac risk assessment and perioperative cardiac management in non-cardiac surgery. Eur Heart J 2009;30(22): 2769-2812. DOI: 10.1093/eurheartj/ehp337.

10. Fleisher LA. Risk of anesthesia. In: Miller RD. ed., Anesthesia 5th ed., Philadelphia: Churchill-Livingstone; 2000. pp. 795-823.

11. Eagle KA, Berger PB, et al. ACC/AHA Guideline, Update for Perioperative Cardiovascular Evaluation for Noncardiac SurgeryExecutive Summary. A report of the American College of Cardiology/ American Heart Association Task Force on Practice Guidelines (Committee to Update the 1996 Guidelines on Perioperative Cardiovascular Evaluation for Noncardiac Surgery). Anesth Analg 2002;94:1052-1064. DOI: 10.1097/00000539-200205000-00002.

12. Warner MA, Offord KP, et al. Role of preoperative cessation of smoking and other factors in postoperative pulmonary complications: a blinded prospective study of coronary artery bypass patients. Mayo ClinProc 1989;64:609-616. DOI: 10.1016/S0025-6196(12) 65337-3.

13. Smetana GW. Current concepts: preoperative pulmonary evaluation. NEngl J Med 1999;340:937-944. DOI: 10.1056/NEJM199903253401207.

14. Rodgers A, Walker N, et al. Reduction of postoperative mortality and morbidity with epidural and spinal anesthesia: Results from overview of randomized trials. BMJ 2000;321:1-12. DOI: 10.1136/ bmj.321.7275.1493. 